\title{
NEW ANALYSIS TO DETERMINE THE MOBILITIES OF CONSTITUENT IONS IN A COMPOSITE MOBILITY SPECTRUM USING DYNAMIC EQUILIBRIUM THEORY
}

\author{
K. Iinuma ${ }^{1)}, \mathrm{T} . \mathrm{Haga}^{2)}, \mathrm{M} . \mathrm{Kagawa}^{3)}$, and S. Kondo ${ }^{4)}$
}

1) Department of Environmental Information Engineering, Tohoku Institute of Technology, Taihaku-ku, Sendai 982-8577, Japan

2) Tokyo Electron AT, Inc., Nirasaki, Yamanashi 407-8511, Japan

3) Daikin Kogyo, Inc., Sakai, Osaka 592-8331, Japan

4) Kyodo Insatsu, Inc., Koishikawa, Tokyo 112-8501, Japan

\begin{abstract}
We have developed a new analysis method, based on the dynamic equilibrium theory, to determine the individual mobilities of component ions constituting a composite mobility spectrum in the atmosphere. Ion Mobility Spectrometer (IMS) gives us the mobilities of component ions and their composite (fake) ion in terms of their ion current (or ion flux) measurements. On the other hand, pure Mass Spectrometer (MS) measurements can determine the ratios of the number densities of component ions in equilibrium. A relatively simple algebraic treatment of these two measurement data allows us to determine the mobilities of all the component ions consisting the composite ion family without any information on their reactions such as reaction frequencies or reaction rate constants. The soundness of this new analysis is examined using the solution of coupled diffusion-reaction equations of four model ions. Then the mobility values of component ions in hydrated ammonium ion family $\left[\left(\mathrm{H}_{2} \mathrm{O}\right)_{\mathrm{n}} \mathrm{NH}_{4}{ }^{+} ; \mathrm{n}=0 \sim 10\right]$ are proposed by analyzing the experimental data of Kim et al. and Nagato, and compared with theoretical values calculated by omega-integral method.
\end{abstract}

Key words: Atmospheric ions, Dynamic equilibrium, Mobility, Ammonium ion family

\section{Introduction}

Theoretical and experimental study on the transport and reaction process of atmospheric ions has a long history because of their important roles in governing the electrical conductivity as well as in encouraging the ion induced nucleation and formation of air pollutants/aerosols in the environmental atmosphere (Holzworth, 1995; Viggiano and Arnold, 1995). In this point of view, the ion mobility is one of the most important coefficients in the field of atmospheric electricity. Nowadays, our common understanding of their nature is that single mobility spectrum reaching equilibrium in the atmosphere consists of a group of several different ionic species with apparently same mobility. This is known as a cluster-ion family. This concept of a composite mobility spectrum has been supported by many experimental findings obtained by laboratory measurements such as drift-tube experiments as well as several field-work-measurements since mid-1970 ${ }^{\text {th }}$ (Mohnen, 1977; Kim et al. 1978; Nagato, 2001). However, the mobilities of individual ions as well as their distribution function are absolutely needed to be determined because of the precise 
information on accurate spatiotemporal variation of atmospheric electrical conductivity. So, the following idea may be attractive: Is it possible to determine separately the mobilities of all the component ions from only one composite mobility spectrum in the atmosphere?

In mid-1990 ${ }^{\text {th }}$, we have obtained a full solution of a coupled parabolic type partial differential equations (coupled diffusion-reaction equations) with respect to the number densities of arbitrary number of ions for the analysis of transport and reactive process of multiple ionic species (Iinuma, 1991; linuma, 1998) in drift-tube experiments (Mason and McDaniel, 1988). It was not long before we have derived an explicit form of an equilibrium solution that describes the state of dynamic balance between the transport process and the reactive process of an arbitrary number of ionic species. (Iinuma et al., 1993). The state of dynamic equilibrium of many positive and negative ionic species is one remarkable characteristics in earth's atmospheric ions, where a unique mobility value consisting of mass-identified many positive and negative ions can experimentally be determined by ion mobility spectrometer associated with mass spectrometer (IMS/MS; Nagato, 2001). It is noted that a series of these data supplies the ratio of the ion currents, namely, the ratio of the products of the drift velocity and the number density of each ionic species in equilibrium. On the other hand, the pure mass spectrometry (MS) applied in equilibrium analysis will provide the ratio of the number densities of many ionic species in the atmosphere. The idea of this research work, based on the dynamic equilibrium theory, is to develop the algebraic method for derivation of all the mobilities of the constituent ions from a composite single mobility value using the IMS/MS data and MS data simultaneously.

\section{Dynamic equilibrium theory}

Various kinds of reactions for innumerable ionic species are occurring ceaselessly in the atmosphere. In general, this kind of particle balance system can be treated by coupled diffusion-reaction equations of many chemical constituents reacting by a pseudo-first order reaction process in a particle conservation system. The following reaction matrix, $A$, is essential for the analysis of this complicated chemical system;

$$
A=\left[\begin{array}{ccccc}
R_{1} & \alpha_{12} & \alpha_{13} & \cdots & \alpha_{1 n} \\
\alpha_{21} & R_{2} & \alpha_{23} & \cdots & \alpha_{2 n} \\
\cdots & \cdots & \cdots & \cdots & \cdots \\
\cdots & \cdots & \cdots & \cdots & \cdots \\
\alpha_{n 1} & \alpha_{n 2} & \alpha_{n 3} & \cdots & R_{n}
\end{array}\right]
$$

where $\alpha_{i j}$ is the reaction frequency per ion from ionic species $j$ to $i$, and

$$
R_{j}=-\sum_{k=1}^{n} \alpha_{k j}
$$

$R_{j}$ expresses a sum of reaction frequencies for all depleting reactions of $j$. It is noted that the determinant of matrix $A$ becomes zero in a particle conservation system. 
In dynamic equilibrium the mobility spectra of all the ionic species become similar Gaussian form with one single mobility value and an equal Full-Width at Half the Maximum (FWHM) (Mohnen, 1977; Kim et al. 1978). This experimental fact was confirmed mathematically by dynamic equilibrium theory developed by us (linuma et al., 1993). In equilibrium the single drift velocity, $v_{s}$, determined from the peak time of single composite mobility spectrum is represented as

$$
v_{s}=\frac{\sum_{j=1}^{n} \delta(j) v(j)}{\sum_{j=1}^{n} \delta(j)}
$$

where $\delta(j)$ is the cofactor of the $(j, j)$ element of the determinant of matrix $A$, and $v(j)$ is the drift velocity of ions $j$. A typical example of arrival time spectra in dynamic equilibrium is shown in Fig.1. These are numerically calculated spectra of four model ions which transport parameters (drift velocities and diffusion coefficients) and mutual reaction frequencies are listed in Table 1. Despite that the drift velocities as well as the diffusion coefficients of four ions are absolutely different values with each other, all the spectra have similar Gaussian form with almost same drift velocity and same FWHM. In Fig.1 the spectrum referred to as "aver." is the averaged spectrum of four ions spectra. The mean drift velocity can be determined from the mean peak time. Note is that the magnitude of FWHM is mainly governed not by their diffusion process, but by mutual reactions which proceed very fast under one atmospheric pressure condition.

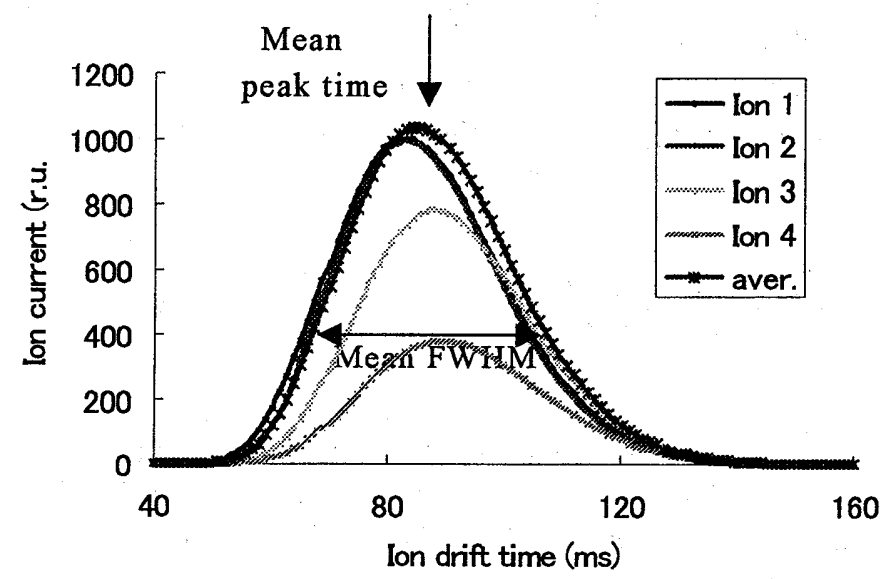

Fig.1 Ion mobility spectra (IMS; arrival time spectra (ATS)) of four model ions in dynamic equilibrium. The drift length is $20 \mathrm{~cm}$. Despite that four model ions have different mobilities and different diffusion coefficients with each other (listed in Table 1), all the spectra form the similar Gaussian shape, gathering together at the same peak position. 
Table 1 Drift velocities $\left(v_{j}\right)$, diffusion coefficients $\left(D_{j}\right)$, and mutual reaction frequencies $\left(\alpha_{i j}\right)$ of four model ions.

\begin{tabular}{|c|c|c|c|c|c|}
\hline \multicolumn{2}{|c|}{} & $j=1$ & 2 & 3 & 4 \\
\hline \multicolumn{2}{|c|}{$v_{j}\left(\mathrm{~cm} \mathrm{~s}^{-1}\right)$} & 400 & 300 & 200 & 100 \\
\hline \multicolumn{2}{|c|}{$D_{j}\left(\mathrm{~cm}^{2} \mathrm{~s}^{-1}\right)$} & 4 & 3 & 2 & 1 \\
\hline \multirow{2}{*}{$\alpha_{i j}$} & $i=1$ & - & 30 & 40 & 42 \\
\cline { 2 - 6 }$\left(\mathrm{s}^{-1}\right)$ & 2 & 36 & - & 30 & 20 \\
\cline { 2 - 6 } & 3 & 60 & 12 & - & 45 \\
\cline { 2 - 6 } & 4 & 70 & 48 & 24 & - \\
\hline
\end{tabular}

Equation (3) stands for an important fact that when the partial pressure of trace gases $\left(\mathrm{H}_{2} \mathrm{O}\right.$, for example) and /or gas temperature changes in the atmosphere, a new equilibrium distribution is established, resulting a different value of mean drift velocity, $v_{s}$ (or mean mobility). In drift tube experiments, the ion mobility spectrum can be detected by the ion current flux (the product of ion number density and its drift velocity) as a function of elapsed time. Then, in dynamic equilibrium we can well approximate the ratio of the areas of the mobility spectra of ions $j$ to $k,\left(S_{j} / S_{k}\right)$, or the ratio of their heights $\left(H_{j} / H_{k}\right)$ using the ratio of the products of their number densities and drift velocities as

$$
\frac{S_{j}}{S_{k}} \cong \frac{H_{j}}{H_{k}} \cong \frac{\delta(j) v(j)}{\delta(k) v(k)}=\frac{N_{e q}(j) v(j)}{N_{e q}(k) v(k)}
$$

where $N_{e q}(j)$ and $N_{e q}(k)$ are the ion number densities of ions $j$ and $k$ in equilibrium. Another important ratio in dynamic equilibrium is the ratio of the ion number densities, which can directly be measured by mass spectrometry (MS). Then, the equilibrium constant of ions $j$ to $k, K_{e q}(j, k)$, can be expressed as

$$
K_{e q}(j, k)=\frac{\delta(j)}{\delta(k)}=\frac{N_{e q}(j)}{N_{e q}(k)}
$$

Figure 2 shows the number densities of four model ions for appearance sake of mass spectra 


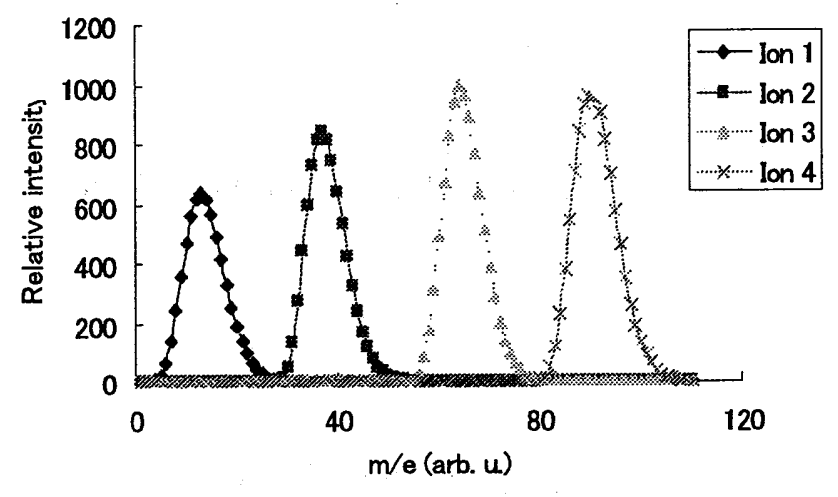

Fig. 2 Mass spectra (MS) of four model ions in dynamic equilibrium. Four spectra show the calculated ion number densities which are modeled on usual mass spectrometry data by separating them relevantly. The ratio of the area of four spectra shows their relative ion number densities.

chart, which are calculated using the transport/reaction coefficients listed in Table 1. The relative number densities of ions can be read out of these spectral heights.

Now, we define two sets of parameters, $X(k)$ and $Y(k)(k=1,2,3, \ldots, \mathrm{n})$, using two measurable values $\delta(k)$ and $v(k)(k=1,2,3, \ldots, \mathrm{n})$ as

$$
\begin{aligned}
& \frac{\delta(k)}{\delta(1)}=X(k) \quad, \quad k=1,2,3, \cdots \cdot, n \\
& \frac{\delta(k) v(k)}{\delta(1) v(1)}=Y(k) \quad, \quad k=1,2,3, \cdots \cdot, n
\end{aligned}
$$

From these parameters and Eq.(3), we can express $v_{s}$ as

$$
v_{s}=\frac{\delta(1) v(1)[Y(1)+Y(2)+Y(3)+\cdots \cdots+Y(n)]}{\delta(1)+\delta(2)+\delta(3)+\cdots \cdots+\delta(n)}
$$

Equation (8) is modified to obtain the drift velocity of ions $1, v(1)$

$$
v(1)=v_{s} \frac{X(1)+X(2)+X(3)+\cdots \cdots+X(n)}{Y(1)+Y(2)+Y(3)+\cdots \cdot+Y(n)}
$$

All the velocities of other constituent ions, $v(k)$, are derived from Eqs. (6) and (7) 


$$
v(k)=v(1) \frac{Y(k)}{X(k)} \quad, \quad k=1,2,3, \cdots \cdot, n
$$

We can replace the drift velocity $v(j)$ with the reduced mobility $K_{0}(j)$ using the following formula,

$$
\left.\left.v(j) \mid c m s^{-1}\right]=26868 \times K_{0}(j) \mid c m^{2} V^{-1} s^{-1}\right] \times E / N[T d]
$$

Then, the mobility $K_{0}(k)$ is represented by $K_{o s}$ in equilibrium as,

$$
\begin{aligned}
& K_{0}(1)=K_{0 S} \frac{X(1)+X(2)+X(3)+\cdots \cdots+X(n)}{Y(1)+Y(2)+Y(3)+\cdots \cdots+Y(n)} \\
& K_{0}(k)=K_{0}(1) \frac{Y(k)}{X(k)}, \quad k=1,2,3, \cdots, n
\end{aligned}
$$

where, $E / N$ in Eq. (11) is the ratio of supplied DC electric field to the number density of ambient gas. The unit is "Td (Townsend)" and $1 \mathrm{Td}=10^{-17} \mathrm{~V} \cdot \mathrm{cm}^{2}$. Equations (12) and (13) are the target formulae of the present research.

\section{Test of present analysis using four model ions}

Now we examine the soundness of this algebraic treatment and Eqs. (12) and (13) using four model ions shown in Fig. 1. Suppose that the reduced electric field is set to be 1Td. Then, the drift velocities in Table 1 and Eq. (11) gives the mobilities of four ions as 1.50 , $1.12,0.74$, and $0.37 \mathrm{~cm}^{2} \mathrm{~V}^{-1} \mathrm{~s}^{-1}$. It is our present interest whether or not the above analysis can reproduce these original mobility values correctly. From Fig. 1 and Eq. (7) we can obtain the following values;

$$
Y(1)=1, \quad Y(2)=0.99, \quad Y(3)=0.78, \quad Y(4)=0.38
$$

Also, from Fig. 2 and Eq. (6),

$$
X(1)=1, \quad X(2)=1.32, \quad X(3)=1.57, \quad X(4)=1.51
$$

The mean reduce mobility in dynamic equilibrium, $K_{0 \text { s }}$, can be determined from Fig. 1 as 


$$
K_{0 s}=\frac{20(\mathrm{~cm})}{86 \times 10^{-3}(\mathrm{~s}) \times 268.68}=0.87\left(\mathrm{~cm}^{2} V^{-1} \mathrm{~s}^{-1}\right)
$$

Using these measurable quantities, we can calculate $K_{0}(1)$ as,

$$
K_{0}(1)=0.87 \times \frac{1+1.32+1.57+1.51}{1+0.99+0.78+0.38}=1.49 \mathrm{~cm}^{2} V^{-1} \mathrm{~s}^{-1}
$$

Then, other three mobilities are derived from $K_{0}(1)$ as

$$
K_{0}(2)=1.49 \times \frac{0.99}{1.32}=1.12, \quad K_{0}(3)=1.49 \times \frac{0.78}{1.57}=0.74, \quad K_{0}(4)=1.49 \times \frac{0.38}{1.51}=0.37
$$

The corresponding original mobility values are $1.50,1.12,0.74$, and $0.37 \mathrm{~cm}^{2} \mathrm{~V}^{-1} \mathrm{~s}^{-1}$, respectively. The agreement between the original value and the calculated value is quite good.

\section{Application to hydrated-ammonium ion family}

Mobility and gas-phase ion chemistry of ammonium ion family is an intriguing research subject, and many research groups have studied the transport/ reaction behavior of this trace gas family using drift tube, flowing after glow experiment, mass spectrometer, plasma chromatography, and IMS/MS (Hogg et al., 1965; Hogg et al., 1966; Fehsenfeld et al., 1973; Carroll et al., 1975; Kim et al. 1978; Eisele, 1989; Nagato et al., 1999; Nagato, 2001). While it is still controversial which ions are the terminal ones in our environmental atmosphere, the ammonium cluster ions, especially hydrated ammonium cluster ions, may be one of the key species because of its relatively stable physical/chemical characteristics. For example, the rate constant of the following exchange reaction;

$$
\mathrm{H}_{3} \mathrm{O}^{+}\left(\mathrm{H}_{2} \mathrm{O}\right)_{n}+\mathrm{NH}_{3} \rightarrow \mathrm{NH}_{4}^{+}\left(\mathrm{H}_{2} \mathrm{O}\right)_{n-1}+2 \mathrm{H}_{2} \mathrm{O}
$$

proposed by Viggiano and Arnold (1995) appears to be relatively large (fast reaction);

$$
k=0.91 \times 10^{-9}(300 / T)^{0.37} \mathrm{~cm}^{3} \mathrm{~s}^{-1}
$$

for $\mathbf{n}=3$, where $T$ is gas temperature in degree Kelvin. Here, we will apply our present analysis to the experiments of hydrated ammonium cluster ions performed by two groups; Kim et al. (1978) and Nagato (2001).

Kim et al. (1978) observed $\left(\mathrm{N}_{2}\right)_{\mathrm{m}}\left(\mathrm{H}_{2} \mathrm{O}\right)_{n} \mathrm{NH}_{4}{ }^{+}$ion family in their plasma chromatography 
experiment. The experimental condition, using $\mathrm{N}_{2}$ as a carrier gas containing $0.03 \% \mathrm{vol}$ of $\mathrm{NH}_{3}$, is as follows; pressure: $762-764$ Torr, temperature: $428 \mathrm{~K}$, electric field: $214-247 \mathrm{~V} \mathrm{~cm}$, drift length: $5.0 \mathrm{~cm}$. The average peak time of single composite mobility spectrum was $5.7 \mathrm{~ms}$. From these values we calculated the $K_{0 s}$ in Eq. (12) as $2.44 \mathrm{~cm}^{2} \mathrm{~V}^{-1} \mathrm{~s}^{-1}$. Also, the values of $Y(k)$ were directly determined from the heights of each mobility spectra as

$$
Y(1)=1, \quad Y(2)=0.17, \quad Y(3)=0.24, \quad Y(4)=0.052, \quad Y(5)=0.14, \quad Y(6)=0.024
$$

where, the ions corresponding to the number 1 to 6 are $\mathrm{NH}_{4}^{+},\left(\mathrm{H}_{2} \mathrm{O}\right) \mathrm{NH}_{4}^{+},\left(\mathrm{N}_{2}\right) \mathrm{NH}_{4}{ }^{+}$, $\left(\mathrm{N}_{2}\right)\left(\mathrm{H}_{2} \mathrm{O}\right) \mathrm{NH}_{4}{ }^{+},\left(\mathrm{N}_{2}\right)_{2} \mathrm{NH}_{4}{ }^{+}$, and $\left(\mathrm{N}_{2}\right)_{3} \mathrm{NH}_{4}{ }^{+}$, respectively. Unfortunately, the values of $X(k)$ were not appeared in their paper. Then, we can resort to a well-known mobility formula derived from molecular kinetics (Mason and McDaniel, 1988); the ion mobility is inversely proportional to the reciprocal of the square root of the reduced mass of an ion and an ambient molecule. Under this assumption we determined $X(k)$ using $\mathrm{N}_{2}$ as an ambient molecule. From Eq.(13),

$$
X(k)=Y(k) \frac{K_{0}(1)}{K_{0}(k)}=Y(k) \sqrt{\frac{\mu(k)}{\mu(1)}}, \quad k=1,2,3, \cdots, n
$$

where, $\mu(k)$ is the reduced mass of a $k$-th ion and a $\mathrm{N}_{2}$ molecule. Then, we can obtain $X(k)$ as

$$
X(1)=1, \quad X(2)=0.20, \quad X(3)=0.30, \quad X(4)=0.069, \quad X(5)=0.19, \quad X(6)=0.034
$$

Substituting $K_{o s}, Y(k)$ and $X(k)$ into Eqs. (12) and (13), we can calculate the mobilities of Kim group, $K_{0}(0)^{\mathrm{a})}$, which are listed in Table2. For mobilities of $\left(\mathrm{H}_{2} \mathrm{O}\right)_{3} \mathrm{NH}_{4}^{+}$and $\left(\mathrm{H}_{2} \mathrm{O}\right)_{5} \mathrm{NH}_{4}^{+}$, these values may somewhat be affected by the clustering reactions involving nitrogen molecules.

Nagato performed IMS/MS experiments and determined the averaged reduced mobility of $\left(\mathrm{H}_{2} \mathrm{O}\right)_{\mathrm{n}} \mathrm{NH}_{4}^{+}$as $1.66 \mathrm{~cm}^{2} \mathrm{~V}^{-1} \mathrm{~s}^{-1}$ (Nagato, 2001). Contrary to Kim's experiment, Nagato' data is only those for the $X(k)$ values and no $Y(k)$ values are available. So, again, we calculated $Y(k)$ from $X(k)$ using Eq. (19). The resulting mobilities, $K_{0}(0)^{\text {b) }}$, are listed in Table 2 .

\section{Discussion}

The present analysis is basically founded on the dynamic equilibrium theory of finite number (four in this case) of model ions. In this limited condition, the analysis appears to be appropriate for ion mobility calculation. However, recent IMS/MS experiments have made clear that innumerable numbers of ionic species are present in natural atmosphere (Eisele, 1989; Viggiano et al., 1995; Nagato, 2001). Therefore, Eqs. (12) and (13) must be generalized as 
Table 2. Mobilities of hydrated ammonium ions $\left(\mathrm{cm}^{2} \mathrm{~V}^{-1} \mathrm{~s}^{-1}\right)$.

a) Kim et al., b) Nagato (1), c) Nagato (2), d) Theoretical

\begin{tabular}{|c|l|c|c|c|c|}
\hline $\mathrm{m} / \mathrm{e}$ & \multicolumn{1}{|c|}{ ion } & $\mathrm{K}_{0}(0)^{\mathrm{a})}$ & $\mathrm{K}_{0}(0)^{\mathrm{b})}$ & $\left.\mathrm{K}_{0}(0)^{\mathrm{c}}\right)$ & $\mathrm{K}_{0}(0)^{\mathrm{d})}$ \\
\hline 18 & $\mathrm{NH}_{4}^{+}$ & 2.69 & 2.05 & 2.26 & 2.93 \\
\hline 36 & $\left(\mathrm{H}_{2} \mathrm{O}\right) \mathrm{NH}_{4}{ }^{+}$ & 2.29 & 1.71 & 1.88 & 2.49 \\
\hline 54 & $\left(\mathrm{H}_{2} \mathrm{O}\right)_{2} \mathrm{NH}_{4}^{+}$ & - & 1.58 & 1.74 & 2.29 \\
\hline 72 & $\left(\mathrm{H}_{2} \mathrm{O}\right)_{3} \mathrm{NH}_{4}^{+}$ & $2.03(?)$ & 1.51 & 1.67 & 2.11 \\
\hline 90 & $\left(\mathrm{H}_{2} \mathrm{O}\right)_{4} \mathrm{NH}_{4}^{+}$ & - & 1.47 & 1.62 & 1.90 \\
\hline 108 & $\left(\mathrm{H}_{2} \mathrm{O}\right)_{5} \mathrm{NH}_{4}^{+}$ & $1.90(?)$ & 1.44 & 1.59 & - \\
\hline 126 & $\left(\mathrm{H}_{2} \mathrm{O}\right)_{6} \mathrm{NH}_{4}^{+}$ & - & 1.42 & 1.57 & - \\
\hline 144 & $\left(\mathrm{H}_{2} \mathrm{O}\right)_{7} \mathrm{NH}_{4}^{+}$ & - & 1.40 & 1.54 & - \\
\hline 162 & $\left(\mathrm{H}_{2} \mathrm{O}\right)_{8} \mathrm{NH}_{4}^{+}$ & - & 1.39 & 1.54 & - \\
\hline 180 & $\left(\mathrm{H}_{2} \mathrm{O}\right)_{9} \mathrm{NH}_{4}^{+}$ & - & 1.37 & 1.52 & - \\
\hline 198 & $\left(\mathrm{H}_{2} \mathrm{O}\right)_{10} \mathrm{NH}_{4}^{+}$ & - & 1.36 & 1.49 & - \\
\hline
\end{tabular}

$$
K(1)=K_{0 s} \times \frac{\sum_{k=1}^{\infty} X(k)}{\sum_{k=1}^{\infty} Y(k)}, K(j)=K(1) \frac{Y(j)}{X(j)} \quad(j=1,2,3, \cdots \cdots)
$$

where the inequality

$$
X(j) \geq Y(j) \quad(j=1,2,3, \cdots \cdots)
$$

will always hold. At present, we cannot calculate the mobilities of all the ions in a composite mobility spectrum because it is impossible to measure experimentally the infinite number of $X(j)$ and $Y(j)$ simultaneously with adequate accuracy. Then, we need some approximation technique for this "infinity problem". According to Nagato's MS data on $\left(\mathrm{H}_{2} \mathrm{O}\right)_{\mathrm{n}} \mathrm{NH}_{4}{ }^{+}$family, the ions which molecular mass is larger than $198(\mathrm{n}=10)$ still exist in this family, though their relative number density is far much smaller than those of small cluster ions. So, for instance, the following assumption may be made to calculate the mobilities of this kind of infinite series of cluster ion family; 


$$
\sum_{k=1}^{\infty} X(k) \approx \sum_{k=1}^{10} X(k)+\eta X(11), \quad \sum_{k=1}^{\infty} Y(k) \approx \sum_{k=1}^{10} Y(k)+\xi Y(11)
$$

where, $\eta$ and $\xi$ are two compensation parameters for residual series of ions in the family. Although we have not yet any appropriate method how to determine these two parameters, we, at present, assume $\eta=10, \xi=10$ by examining the tendency of the number densities of the series of ions in Nagato's real MS data. Under this assumption, we can calculate the mobilities, $K_{0}(0)^{\text {c) }}$, which are listed in Table 2. Theoretical mobilities, $K_{0}(0)^{\text {d) }}$, derived using omega-integral method are also listed in it (Iinuma et al., 1997). For $\mathrm{NH}_{4}^{+}$ion, the mobility of $2.69 \mathrm{~cm}^{2} \mathrm{~V}^{-1} \mathrm{~s}^{-1}$ calculated from Kim group data is larger than those of Nagato's data, $2.05 \mathrm{~cm}^{2} \mathrm{~V}^{-1} \mathrm{~s}^{-1}$ (11-ionic species analysis) and $2.26 \mathrm{~cm}^{2} \mathrm{~V}^{-1} \mathrm{~s}^{-1}$ (compensation parameter analysis). This discrepancy may attributed to the difficulty of ion swarm experiments in dynamic equilibrium, in which the reproducibility of $K_{0 s}$ measurement may not be so good. In addition, measurement of $Y(k)$ may need a very accurate ion current detection or counting technique of low number density ions. On the other hand, the reason of large theoretical mobility (Iinuma et al., 1997) may be caused by the usage of rather simple potential model (point-charge-potential-model) in omega-integral calculation.

While the present method has a potential to be useful for determination of mobilities of a composite ion family with adequate accuracy, one basic problem is how we can confirm the dynamic equilibrium state of reactive transport phenomena of multiple ions. One qualitative confirmation is to check the tendency for all the ATS to gather at the same peak time in IMS experiment. In addition, we should be careful all the ATS profiles must approach the symmetric Gaussian shapes with the same FWHM. Comparison of the ATS measured at several different drift lengths may be much more useful to confirm the attainment of symmetric Gaussian shape. No researches for more quantitative analysis of the attainment of dynamic equilibrium are developed yet.

Also, it is still controversial what is the final terminal products in the atmosphere; ammonium-family, pyridine, or amine? According to Nagato's MS experiment (Nagato, 2001), a series of protonated alkyl-substituted pyridines and amines were observed which might be two major candidates of terminal ion families because of their higher proton affinity than that of $(\mathrm{OH})_{\mathrm{n}} \mathrm{NH}_{3}$. However, the presence of atmospheric chemicals (and ions) and their spatiotemporal evolution depends strongly on the atmospheric (experimental) conditions; the temperature, the humidity, and the concentrations of trace gas compositions such as ammonia, sulfur compounds, nitrogen compounds, and various kinds of natural and anthropogenic VOCs. Because of these time/area dependent conditions, the ion mobility distribution will ceaselessly change from time to time and area to area. So, rigorously, IMS data and MS data measured at different time/area (or different experimental conditions) should not be combined together in the present dynamic equilibrium analysis. In this point, the relatively large errors of the estimated mobility values seem to be unavoidable in the present analysis of hydrated-ammonium ion family based on Kim's data and Nagato's data. However, emphasized in the present analysis is that the dynamic equilibrium state contains not only the information on reaction rates but also the information on the mobilities (and also diffusion coefficients) of all ionic species in a reactive-transport system. If, in dynamic equilibrium state, IMS experiments can measure separately all the ATSs of 
constituent ions one by one, and if the corresponding MS data can assign them correctly, we can determine the accurate values of mobilities of all the ionic species irrelevant to their identity. These correct mobility values and its distribution will be very helpful to estimate the spatiotemporal variation of the electrical conductivity of the atmosphere and to reveal the cause and effect of its geophysical phenomena.

\section{Conclusions}

A new analysis method to determine the individual mobilities of atmospheric ion family in a composite mobility spectrum has been developed. Its soundness has been confirmed using a composite mobility spectrum of four model ions. Combining the IMS data and the MS data of eleven hydrated-ammonium ion family measured by two different groups, we have simultaneously derived all the mobilities and compared them with theoretical values.

\section{Acknowledgments}

The authors wish to express their appreciation to Dr. N. Sasaki for his helpful suggestions.

\section{REFERENCES}

Carroll, D. I., I. Dzidic, R. N. Stillwell, and E. C. Horning, Identification of positive reactant ions observed for nitrogen carrier gas in plasma chromatograph mobility studies, Anal. Chem., 47, 1956-1959, 1975.

Eisele, F. L., Natural and transmission line produced positive ions, J. Geophys. Res., 94, 6309-6318, 1989.

Fehsenfeld, F. C. and E. E. Ferguson, Thermal energy positive ion reactions in a wet atmosphere containing ammonia, J. Chem. Phys., 59, 6272-6276, 1973.

Hogg, A. M. and P. Kebarle, Mass-spectrometric study of ions at near-atmospheric pressure. II. Ammonium ions produced by the alpha radiolysis of ammonia and their solvation in the gas phase by ammonia and water molecules, J. Chem. Phys., 43, 449-456, 1965.

Hogg, A. M., R. M. Haynes, and P. Kebarle, Ion-solvent molecule interactions studied in the gas phase. Heats and entropies of individual steps. $\mathrm{NH}_{4}{ }^{+} \cdot(\mathrm{n}-1) \mathrm{NH}_{3}+\mathrm{NH}_{3}=\mathrm{NH}_{4}{ }^{+} \cdot \mathrm{nNH}_{3}$, J. Am. Chem. Soc, 88, 28-31, 1966.

Holzworth, R. H., "Quasistatic Electromagnetic Phenomena in the Atmosphere and Ionosphere", Hans Volland, Ed., Handbook of Atmospheric Electrodynamics, Vol. I, pp233-266, CRC Press, 1995.

Iinuma, K., Analysis of reactive ion transport in weakly ionized gas mixtures, Can. J. Chem., 69, 1090-1099, 1991.

Iinuma, K., N. Sasaki, and M. Takebe, A general analysis of reactive ion transport in dynamic equilibrium, J. Chem. Phys. 99, 6907-6914, 1993.

Iinuma, $\mathrm{K}$. and $\mathrm{H}$. Nishikatsu, A model calculation of transport coefficient for small positive ions in the troposphere, J. Atmos. Electr. 17, 11-22, 1997.

Iinuma, K., A general solution of coupled diffusion-reaction equations for the analysis of atmospheric ion chemistry in particle non-conservation systems, J. Atmos. Electr. 18, 119-124, 1998. 
Kim, S. H., K. R. Betty, and F. W. Karasek, Mobility behavior and composition of hydrated positive reactant ions in plasma chromatography with nitrogen carrier gas, Anal. Chem., 50, 2006-2012, 1978.

Mason, E. A. and E. W. McDaniel, Transport Properties of Ions in Gases, Wiley, New York, 1988.

Mohnen, V. A., "Formation, nature, and mobility of ions of atmospheric importance", in Electrical Processes in Atmospheres, 1-16, Steinkopf, Darmstadt, Germany, 1977.

Nagato, K., D. J. Tanner, H. R. Friedli, and F. L. Eisele, Field measurement of positive ion mobility and mass spectra at a Colorado site in winter, J. Geophys. Res., 104, 3471-3482, 1999.

Nagato, K., Development and evaluation of an ion mobility spectrometer/mass spectrometer (IMS/MS) for the analysis of ion-molecule reactions at atmospheric pressure, J. Atmos. Electr., 21, 31-47, 2001.

Viggiano, A. A., and F. Arnold, "Ion Chemistry and Composition of the Atmosphere", Hans Volland, Ed., Handbook of Atmospheric Electrodynamics, Vol. I, pp1-25, CRC Press, 1995.

(Received December 5, 2005; revised January 15, 2006; accepted February 18, 2006) 